\title{
Transtorno do Déficit de Atenção/Hiperatividade: o que nos informa a investigação dimensional?
}

\author{
Alana Concesso Andrade \\ Carmen Flores-Mendoza \\ Universidade Federal de Minas Gerais
}

\begin{abstract}
Resumo
Investiga-se o Transtorno do Déficit de Atenção/Hiperatividade (TDAH) em contexto escolar adotando a perspectiva dimensional. Participaram 107 crianças com idade média de 12,37 anos. Utilizou-se a Escala de TDAH - versão para professores, uma versão adaptada para pais e tarefas computadorizadas de processamento cognitivo. A prevalência de Hiperatividade pelo relato de professores e pais foi condizente com aquela apontada pela literatura (4,7\%). O Déficit de Atenção, avaliado por pais e professores, correlacionou com todas as medidas de processamento de informação. Hiperatividade e Comportamento Anti-social correlacionaram com as medidas de memória de trabalho quando a primeira foi avaliada pelos professores e a segunda pelos pais, refletindo baixa concordância entre o relato de informantes a respeito das dimensões comportamentais do TDAH. Entretanto, as respostas dos professores parecem estar mais de acordo com a literatura. Conclui-se que a perspectiva dimensional de investigação pode fornecer informações relevantes sobre as características demográficas e cognitivas do TDAH.
\end{abstract}

Palavras-chave: hiperatividade; déficit de atenção; prevalência; diferenças individuais; processamento cognitivo.

\begin{abstract}
Attention Deficit/Hyperactivity Disorder: what does the dimensional research inform us? This research investigates the Attention Deficit/Hyperactivity Disorder (ADHD) in a school context from a dimensional perspective. It took part of the study 107 children with mean age of 12,37 years. It was used the ADHD's Scale - teacher's version and an adapted form for parents in addition to computer tasks to measure cognitive processing. The prevalence of Hyperactivity, according to teachers and parent's report, was consistent with the literature (4,7\%). Attention deficit correlated with all measures of cognitive processing. Hyperactivity and Antisocial Behavior correlated with working memory measures only when the first was assessed by teachers and the second was assessed by parents. In this sense, it was found low concordance between informants regarding ADHD behavior dimensions. However, teacher's report seems to be according with contemporary literature. It was concluded that dimensional investigation can provides relevant information related to demographic and cognitive characteristic of ADHD.
\end{abstract}

Keywords: hyperactivity; attention deficit; prevalence; individual differences; cognitive processing.

A Hiperatividade com Déficit de Atenção é um dos transtornos que mais acomete a infância e a adolescência. Os sintomas, quando intensificados por falhas de diagnóstico e de tratamento, podem ocasionar fracasso escolar e comportamento anti-social (Barkley, 2002; Manuzza, Klein, \& Moulton, 2002).

Embora seja um transtorno recorrente e de conseqüências individuais e sociais negativas, sua investigação e determinação epidemiológica são buscadas por meio de métodos avaliativos e informantes diversos, o que não fornece dados precisos sobre sua prevalência na população geral. Nos últimos anos, tem sido bastante ressaltada a criação de testes psicológicos e neuropsicológicos para a avaliação no próprio indivíduo com suspeita de TDAH, de forma que não se dependa tanto de dados discordantes de múltiplos informantes.

Tais instrumentos, no entanto, têm trazido baixa validade de critério externo. Alguns autores (Ávila, Cuenca, Felix, Parcet, \& Miranda, 2004) discutem as razões para tanto e focam em duas explicações principais: uma delas se refere a uma indefinição dos comportamentos a serem avaliados pelas provas (por exemplo, deve ser medida a atenção sustentada ou a seletiva?), os quais não refletem adequadamente as características do transtorno. A 
segunda explicação se remete à capacidade que a criança possui, mesmo tendo TDAH, de conter os sintomas durante os poucos minutos em que realiza as tarefas no computador, não sendo possível, portanto, detectar suas dificuldades comportamentais e cognitivas.

Há que se acrescentar que, de maneira geral, atividades feitas no computador são atraentes para a maioria das crianças, as quais se empenham em realizá-las sem que para isso imprimam um esforço adicional. Todavia, é necessário notar que entre os instrumentos informatizados mais promissores para avaliar o TDAH, encontram-se aqueles referentes ao processamento cognitivo como o da avaliação da memória de trabalho (Oosterlaan, Scheres, \& Sergeant, 2005).

Nesse sentido, é importante ressaltar que a literatura do TDAH é rica em dados empíricos, porém razoavelmente pobre em seu embasamento teórico. Dentre as tentativas de erigir uma teoria do transtorno destaca-se a de Barkley (1997) que define o TDAH como um déficit na função inibitória. Existiriam, de acordo com o modelo teórico, quatro funções executivas afetadas pelo déficit: a) memória de trabalho, b) auto-regulação do afeto, c) internalização do discurso e, d) reconstituição (análise e síntese comportamental).

A primeira delas, a memória de trabalho, refere à capacidade de manter um item de informação na mente na ausência de pistas externas para tal e de usar essa informação para dirigir uma resposta iminente. A segunda é a auto-regulação do afeto que se refere à separação da carga emocional do conteúdo de um evento, proporcionando o controle do comportamento motor. A terceira função é a internalização do discurso a qual permite, durante o adiamento do processo decisório da resposta (delay), que o sujeito converse consigo mesmo para gerar instruções autodirigidas fundamentais para o autocontrole. A reconstituição é a quarta função executiva relacionada ao controle inibitório. Por intermédio dela, realiza-se análise e síntese de mensagens e eventos que facilitam o processamento de informação.

A criança hiperativa/impulsiva, portanto, demonstraria uma inibição pobre em todos os comportamentos em que há possibilidade de recompensa imediata do meio (Nigg, 2001). Por essa razão é superativa, fala demais, fala alto consigo mesma, tem dificuldade de restringir o comportamento de acordo com a instrução, resistir à tentação e adiar a gratificação.

O modelo teórico de Barkley explica os processos envolvidos na manifestação da impulsividade. Entretanto, na realização do diagnóstico são utilizadas as recomendações do DSM IV TR (Diagnostic and Statistical Manual da Associação Psiquiátrica Americana - American Psychiatric Association [APA], 2000) que, embora amplamente adotadas e extremamente úteis, deixam algumas dúvidas. Por exemplo, o problema de determinar a manifestação dos sintomas, de forma igualmente prejudicial, em dois contextos comuns à criança. Nesse sentido, considerando-se que escola e lar são os dois contextos mais comuns à criança, a determinação da consistência e intensidade dos sintomas se torna controversa pela quase impossibilidade de encontrar concordância entre pais e professores na avaliação comportamental de uma criança (Crystal, Ostrander, Chen, \& August, 2001; Rowland et al., 2008).

Homer et al. (2000) informam que a discrepância de relatos de pais e professores ocorre devido às expectativas diferentes em relação ao comportamento em casa e na escola e às estratégias diversas adotadas para lidar com esse comportamento e/ou com as circunstâncias do ambiente. Zucker, M. Morris, Ingram, R.D. Morris, e Bakeman (2002) asseveram que a natureza do sintoma avaliado é subjetiva, então, sua intensidade, duração e freqüência são julgadas de formas diferentes. A discordância refletiria atitudes, julgamentos e interpretações divergentes dos itens de uma dada escala e do comportamento em si, além dos possíveis vieses do adulto em relação ao sujeito avaliado.

Além dos vieses relacionados à fonte e às expectativas de comportamento, a discordância entre informantes encontrada na literatura também pode se dever às diferentes metodologias de avaliação empregadas com pais e professores, assim como às restrições amostrais, visto que a já reduzida população clínica é subdividida entre subtipos - predominantemente desatento; predominantemente hiperativo, e combinado (Crystal et al., 2001; Mitsis, McKay, Schultz, Newcorn, \& Halperin, 2000).

Por outro lado é apontado freqüentemente que os pais podem ser fontes confiáveis para diagnóstico de Hiperatividade (Simonsen \& Bullis, 2007), a qual tem uma relação com o comportamento global da criança. Os professores, por sua vez, predizem melhor a Desatenção, cuja associação com os problemas escolares é reconhecida. Dessa forma, o relato das duas fontes se torna aconselhável (Simonsen \& Bullis, 2007; Powers et al., 1998).

Outra ponderação claramente relevante se refere à maior incidência de TDAH entre os indivíduos do sexo masculino. O próprio DSM aponta uma proporção de quatro meninos para cada menina e tal prevalência encontra ecos em diversos estudos (Burns, Walsh, Gomez, \& Hafetz, 2006; Manuzza, Klein, Blesser, Malloy, \& LaPadula, 1998).

De acordo com Barkley (1999), o sexo masculino é mais suscetível a transtornos do Sistema Nervoso Central, como aqueles relacionados ao comportamento impulsivo, e, portanto, à Hiperatividade. Já nas meninas, predominaria o Déficit de Atenção. No entanto, pesquisas atuais não comprovam essa hipótese, uma vez que a incidência é maior no sexo masculino para os três subtipos, de acordo com diversas investigações (Burns et al., 2006; Chhabildas, Pennington, \& Willcut, 2001; Huang-Pollock, Higg, \& Carr, 2005).

Dada esta incerteza delineada pela literatura é relevante apoiar estudos dimensionais a respeito do transtorno. Em outras palavras, deve-se entender que um mesmo transtorno pode apresentar diferentes dimensões que variam, em intensidade, ao longo de um continuum psicológico. No caso do TDAH, as dimensões Hiperatividade e Desatenção são entendidas como correlacionadas entre si, porém associadas a prejuízos distintos.

É cabível observar que a literatura a respeito do TDAH traz diversas problematizações. Nesse sentido, pretendeu-se, no presente estudo, por meio de perspectiva dimensional, determinar: 1) diferenças de sexo na apresentação das características do transtorno; 2) correspondência entre as dimensões avaliadas e o desempenho cognitivo em provas computadorizadas e; 3 ) verificar o grau de concordância entre avaliações de professores e pais. 


\section{Método}

\section{Participantes}

Os participantes desta amostra fazem parte do projeto "Estudo Longitudinal das Competências Psicológicas de Crianças Escolares", desenvolvido através de uma parceria entre o Laboratório de Avaliação das Diferenças Individuais - LADI/ UFMG e o Centro Pedagógico (CP) da UFMG. Essa avaliação consiste na aplicação de uma bateria de testes psicológicos e questionários através dos quais são medidos aspectos ou traços diversificados de uma mesma criança.

No ano de 2005, os professores da escola avaliaram as características do TDAH de 135 crianças das $5^{\underline{a}}, 6^{\underline{a}}$ e $7^{\underline{a}}$ séries do Ensino Fundamental. O sexo masculino representou 55\% da amostra e a média de idade foi de 12,37 com desvio-padrão de 0,78. Não houve na amostra nenhuma criança repetente. Mediante a aplicação do Critério Brasil (Associação Brasileira de Empresas de Pesquisa [ABEP], 2003), as classes econômicas da amostra ficaram representadas em: A2 $(8,2 \%)$, B1 $(11,1 \%)$, B2 $(28,1 \%), C$ (43\%) e D (7,4\%). Não responderam, 2,2\%.

\section{Instrumentos}

Escala de TDAH - Versão para Professores (Benczik, 2000). A escala foi criada e validada a partir da necessidade de um instrumento brasileiro em que professores avaliassem os sintomas do TDAH em contexto escolar. O instrumento foi desenvolvido com base nos critérios do DSM-IV. Nesse sentido, além dos sintomas de Hiperatividade e Déficit de Atenção, o teste também avalia os prováveis prejuízos escolares e sociais apontados como condição diagnóstica pela APA.

A escala é composta de 49 itens distribuídos em quatro sub-escalas: 1) Déficit de Atenção (DA); 2) Hiperatividade/ Impulsividade (HI); 3) Problemas de Aprendizagem (PA) e; 4) Comportamento Anti-social (AS). Os índices Alpha de Cronbach encontrados foram 0,97 para a sub-escala de DA, 0,95 para HI, 0,94 para PA e 0,90 para AS.

Escala de TDAH adaptada para Pais. A fim de comparar os sintomas de TDAH avaliados pelos professores, adaptou-se a mesma escala para os pais. Modificações foram realizadas em quatro itens da sub-escala de Déficit de Atenção, em dois itens de Hiperatividade/Impulsividade e em quatro itens de Comportamento Anti-social. A sub-escala de Problemas de Aprendizagem não foi adaptada para os pais, uma vez que se trata de comportamentos específicos do contexto escolar.

Tarefa informatizada de processamento cognitivo básico - Discriminação Perceptual. Esta prova avalia processamento cognitivo básico e é parte de uma bateria de 12 testes de avaliação cognitiva desenvolvida por Detterman et al. (1992) e adaptada por Flores-Mendoza (1999).

Especificamente, a tarefa chamada de Discriminação consiste da apresentação simultânea de uma seqüência de sete estímulos na parte inferior da tela do computador, de uma janelateste em branco acima dessa seqüência e de um botão de início abaixo da fileira de sete estímulos. Solicita-se do sujeito que identifique rapidamente entre os sete estímulos aquele idêntico, escolhido pelo programa, ao que aparece acima. No momento em que deixa de clicar o botão de início, os estímulos em fileira ficam em branco. Após clique correto ou incorreto, o estímulo correto pisca na tela. A tarefa compreende 168 ensaios (24 estímulos $\mathrm{x} 7$ posições).

A escolha desta tarefa deve-se ao fato de poder verificar, em uma tarefa simples que não demanda processos cognitivos superiores, se as diferenças em tempo de decisão e velocidade de processamento entre os sujeitos estão relacionadas às características do transtorno. Acredita-se, que em tarefas como esta, o déficit de atenção/hiperatividade pode levar a um tempo de reação mais lento e a um maior número de erros (Barkley, 1997, 1999; Derefinko et al. 2008; Quay, 1997).

Tarefa informatizada de memória de trabalho - Alfabeto. Esta tarefa avalia a memória de trabalho e foi desenvolvida por Craik em 1986, sendo adaptada por Flores-Mendoza, Colom, Garcia e Castilho (2001).

O teste denominado Alfabeto consiste na apresentação, na tela do computador, de quatro e cinco palavras, uma de cada vez, por 1,5 segundo, das quais o sujeito deve memorizar as primeiras letras. Ao final da apresentação surge outra tela com quatro ou cinco lacunas nas quais se devem digitar as primeiras letras das palavras mostradas, porém elas devem ser ordenadas alfabeticamente. $\mathrm{O}$ sujeito tem 30 segundos para realizar cada ensaio da tarefa.

Tem-se um total de 30 ensaios, sendo 15 com quatro palavras e outras 15 com cinco palavras. A opção por este tipo de prova computadorizada vem da possibilidade de se observar dificuldades em memória de trabalho apontadas pela literatura como relacionadas aos sintomas do TDAH (Barkley, 1997).

\section{Procedimento}

Primeiramente, as crianças foram autorizadas pelos pais a participar da pesquisa, os quais responderam o questionário Critério Brasil (ABEP, 2003). Após o consentimento, pais e professores avaliaram as crianças por meio da Escala do TDAH - Versão para professores (Benczik, 2000) e versão adaptada.

Especificamente no contexto escolar, as escalas de TDAH foram distribuídas aos professores das $5^{\mathrm{a}}, 6^{\mathrm{a}}$ e $7^{\mathrm{a}}$ séries. No CP existem três turmas para cada série e cada uma dessas turmas possui um professor representante ou "referência", conforme são chamados na escola. Esses professores foram os respondentes das escalas. Anteriormente, lhes foi endereçada uma carta com explicações detalhadas da pesquisa e solicitação de colaboração.

Enquanto os professores avaliaram 135 crianças, os pais remeteram as avaliações de 107 alunos. As análises, portanto, foram realizadas com base nos 107 protocolos.

As tarefas computadorizadas foram aplicadas às crianças, individualmente, em uma sala reservada e silenciosa dentro da própria escola.

\section{Resultados}

\section{Prevalência}

Para a determinação da porcentagem de crianças com altos resultados na avaliação do TDAH, observou-se a freqüência a 
partir do percentil 95, uma vez que, de acordo com o manual de interpretação da Escala do TDAH, é a "região onde há maior probabilidade de apresentar o transtorno" (Benczik, 2000, p. 56). Para tanto, os escores brutos da avaliação de pais e de professores foram transformados em valores percentílicos $(n=107)$. As porcentagens encontradas foram as mesmas para professores e pais: $3,7 \%$ para DA $(n=4)$; e $4,7 \%$ tanto para $\mathrm{HI}$ quanto para $\mathrm{AS}$ ( $n=5$ para cada dimensão).

\section{Diferenças de Sexo}

Para verificar as diferenças de sexo (variável independente) nas pontuações das sub-escalas do TDAH (variável dependente), empregou-se a análise de variância (ANOVA) tipo intergrupos one-way no estudo de protocolos respondidos pelos professores e pelos pais $(n=107)$, sendo 48 crianças do sexo masculino e 59 do sexo feminino. Na Tabela 1 apresentam-se as estatísticas descritivas.

Tabela 1

Estatísticas descritivas das sub-escalas do TDAH considerando sexo

\begin{tabular}{ccccccc}
\hline $\begin{array}{c}\text { Sub- } \\
\text { escalas }\end{array}$ & $\begin{array}{c}\text { Relato } \\
\text { professores } \\
\text { Meninos }\end{array}$ & $\begin{array}{c}\text { Relato } \\
\text { professores } \\
\text { Meninas }\end{array}$ & $F(1,134)$ & $\begin{array}{c}\text { Relato pais } \\
\text { Meninos }\end{array}$ & $\begin{array}{c}\text { Relato pais } \\
\text { Meninas }\end{array}$ & $F(1,000)$ \\
\hline \multicolumn{1}{c}{$M(D P)$} & $M(D P)$ & & $M(D P)$ & $M(D P)$ & \\
DA & $49,37(16,31)$ & $36,41(12,32)$ & $23,16^{* *}$ & $43,74(13,33)$ & $34,58(10,11)$ & $17,45^{*}$ \\
HI & $31,94(12,02)$ & $24,35(9,06)$ & $19,55^{* *}$ & $35,44(12,26)$ & $32,87(11,87)$ & 0,91 \\
AS & $16,59 \quad(5,36)$ & $13,08(3,86)$ & $9,66^{*}$ & $14,55(5,23)$ & $13,12(4,17)$ & 2,38 \\
\hline$*_{p<0,05 * * p<0,001}$ & & & & &
\end{tabular}

Para o relato de professores, a ANOVA dos resultados mostrou haver um efeito principal significativo da variável sexo na sub-escala Déficit de Atenção $[F(1,134)=23,16 ; p=0,000]$, na sub-escala de Hiperatividade $[F(1,134)=19,55 ; p=0,000]$ e na sub-escala de Comportamento Anti-social $[F(1,134)=9,66$; $p=0,002]$. O tamanho do efeito $\eta^{2}$ foi de 0,148 para Déficit de Atenção (efeito considerável); 0,128 para Hiperatividade (efeito moderado) e de 0,068 para Comportamento Anti-social (efeito pequeno). A observação das estatísticas descritivas aponta as pontuações dos meninos como significativamente mais altas.

No caso do relato dos pais, a única diferença estatisticamente significativa entre os sexos aconteceu na sub-escala de Desatenção $[F(1,000)=17,45 ; p=0,000]$. O $\eta^{2}$ foi de 0,142 (efeito considerável) e novamente o sexo masculino apresentou a média mais alta.

\section{Processamento de Informação}

A seguir, analisou-se a correspondência entre as características do TDAH avaliadas por pais e pelos professores e os parâmetros medidos pelas tarefas informatizadas de memória de trabalho e processamento cognitivo simples. Nesse sentido, selecionou-se aleatoriamente uma sub-amostra de 68 crianças, sendo 32 meninas e 36 meninos. A disparidade de sexo ocorreu devido a diferenças na disponibilidade de participação das crianças. Realizou-se correlação parcial com controle da variável sexo, haja vista a tendência dos meninos a apresentarem maior escore no TDAH que as meninas. Na Tabela 2, encontram-se os resultados obtidos com o relato de professores e pais.

Tabela 2

Correlações entre TDAH e variáveis de processamento cognitivo

\begin{tabular}{ccccccc}
\hline Critérios & TD_Disc & TD_MT & Err_Disc & Err_MT & Porc_4MT & Porc_5MT \\
\hline \multirow{2}{*}{ DA } & $0,401^{* *}$ & $0,417^{* *}$ & $-0,194$ & $0,446^{* *}$ & $-0,432^{* *}$ & $-0,438^{* *}$ \\
& $\left(0,291^{*}\right)$ & $\left(0,275^{*}\right)$ & $(-0,085)$ & $\left(0,330^{* *}\right)$ & $\left(-0,345^{* *}\right)$ & $\left(-0,284^{*}\right)$ \\
\hline \multirow{2}{*}{ HI } & 0,206 & $0,333^{*}$ & $-0,085$ & $0,304^{*}$ & $-0,243^{*}$ & $-0,282^{*}$ \\
& $(0,186)$ & $\left(0,229^{*}\right)$ & $(-0,021)$ & $(0,075)$ & $(-0,185)$ & $(-0,150)$ \\
\hline \multirow{2}{*}{ AS } & 0,079 & 0,099 & $-0,047$ & $0,270^{*}$ & $-0,127$ & $-0,178$ \\
& $\left(0,282^{*}\right)$ & $\left(0,313^{*}\right)$ & $(-0,178)$ & $\left(0,259^{*}\right)$ & $\left(-0,350^{* *}\right)$ & $\left(-0,309^{*}\right)$ \\
\hline
\end{tabular}

Nota: Correlações parciais com controle de sexo. Entre parêntese correlações para pais. DA=Déficit de Atenção; $\mathrm{HI}=$ Hiperatividade; $\mathrm{AS}=$ Comportamento Anti-social; TD Disc=Tempo de decisão na tarefa de discriminação TD_MT= Tempo de decisão na tarefa de memória de trabalho; Err_Disc=Número de erros na tarefa de discriminação; Err_MT= Número de erros na tarefa de memória de trabalho; Porc_4MT= Porcentagem de acertos na tarefa de memória de trabalho com 4 letras; Porc_5MT=Porcentagem de acertos na tarefa de memória de trabalho com 5 letras. ${ }^{*} p<0,05 * * p<0,001$

A matriz de correlações permite observar que a dimensão Déficit de Atenção se correlaciona com quase todas as medidas de processamento de informação. A dimensão Hiperatividade, por sua vez, não está associada com processamento básico (tarefa de discriminação), mas sim com processamento complexo (tarefa de memória de trabalho). Neste último, observa-se que quanto maior é o escore em Hiperatividade menor é a velocidade mental (altos tempos de decisão) e menor também é a precisão (maior número de erros). No caso de Comportamento Anti-social, observa-se haver associação negativa significativa apenas com o número de erros na tarefa de memória de trabalho.

Novamente, o mesmo procedimento se aplicou ao relato dos pais. Observa-se na Tabela 2 que, igualmente, a dimensão Déficit de Atenção foi a que mais se associou aos resultados nas tarefas, 
embora os índices tenham sido mais baixos que na avaliação dos professores. A Hiperatividade correlacionou somente com o tempo de decisão em memória de trabalho, ao passo que o Comportamento Anti-social correlacionou com diversas medidas das tarefas, com exceção do número de erros em Discriminação. É interessante enfatizar que o padrão encontrado para professores foi inverso, ou seja, a avaliação do Comportamento Anti-social não apresentou correlações significativas com as tarefas, mas a Hiperatividade sim. Esse resultado parece mostrar que as duas dificuldades comportamentais são discernidas de forma distinta pelos avaliadores.

As análises apresentadas até então mostraram que, aparentemente, existem diferenças na avaliação de pais e professores a respeito das características do transtorno em questão. Por exemplo, para os pais, diferenças significativas entre meninos e meninas somente existem para a Desatenção, enquanto que, para os professores, as diferenças de sexo foram notadas nas três escalas do TDAH. Por outro lado, pais e professores diferem levemente quanto à associação entre TDAH e as tarefas informatizadas.

\section{Concordância entre avaliadores (pais e professores)}

Existem duas questões independentes que rodeiam a comparação de pais e professores sobre como avaliam o comportamento infantil. Uma delas diz respeito ao grau de similaridade em que pais e professores avaliam as crianças, a outra diz respeito a se os pais conseguem avaliar os mesmos construtos que os professores estão avaliando. Dito de outra forma: na primeira questão trata-se de saber, por exemplo, se as crianças que obtiveram alta pontuação no relato de professores recebem também alta pontuação de acordo com os pais. Outra questão é saber se o instrumento utilizado, a Escala do TDAH, foi utilizado de forma equivalente pelos pais e pelos professores.

Para responder à primeira questão, utiliza-se apenas a correlação de Pearson, enquanto que para responder à segunda questão, utiliza-se o Índice de Congruência Fatorial. Uma amostra de 107 crianças avaliadas por pais e professores foi analisada com base nessas duas técnicas.

O Índice de Congruência Fatorial identifica se a escala, apesar de suas leves modificações para pais, foi utilizada de forma semelhante para ambos os grupos de avaliadores. Isto é, talvez a baixa concordância em aspectos comportamentais deva-se a que pais e professores apresentam conhecimento diferenciado na avaliação dos itens. Por exemplo, os pais podem ser mais ou menos condescendentes na avaliação de itens como "Age imprudentemente" (item 23 de Hiperatividade) ou "Respeita as regras dadas" (item 48 de Comportamento Anti-social).

A técnica que permite identificar se os construtos latentes refletidos na estrutura fatorial da Escala do TDAH, a partir das respostas de pais e professores, são, de fato, congruentes chamase Índice de Congruência Fatorial (Jensen, 1998) e é determinado através da seguinte equação:

Na Equação 1, CCpq é o coeficiente de congruência, $n$ é o número de variáveis nas duas amostras (pais e professores), $p$ é número de fatores no primeiro conjunto de variáveis (pais), $q$ é o número de fatores no segundo conjunto de variáveis (professores), $S 1$ é a carga fatorial no primeiro conjunto e $S 2$ é

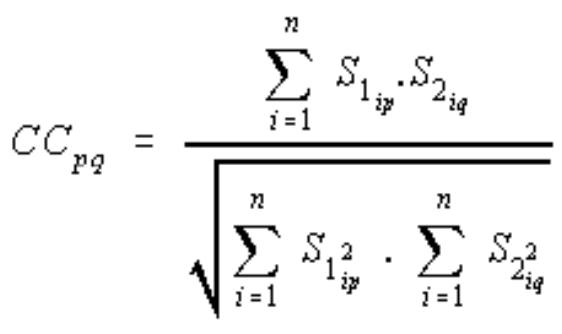

a carga fatorial no segundo conjunto.

Um índice de concordância considerado como de "razoável similaridade fatorial" é de 0,90 e como "altamente similar ou praticamente idêntica" é o valor acima de 0,95 (Jensen, 1998). Os resultados referentes à análise fatorial exploratória com rotação varimax das respostas de pais e professores, permitem inferir que estes últimos avaliaram de forma razoavelmente similar o Déficit de Atenção e a Hiperatividade, mas no caso da avaliação de Comportamento Anti-social, pais e professores parecem ter critérios diferenciados. A Tabela 3 apresenta resultados de correlação da pontuação bruta nas escalas respondidas por pais e professores e também o Índice de Congruência Fatorial.

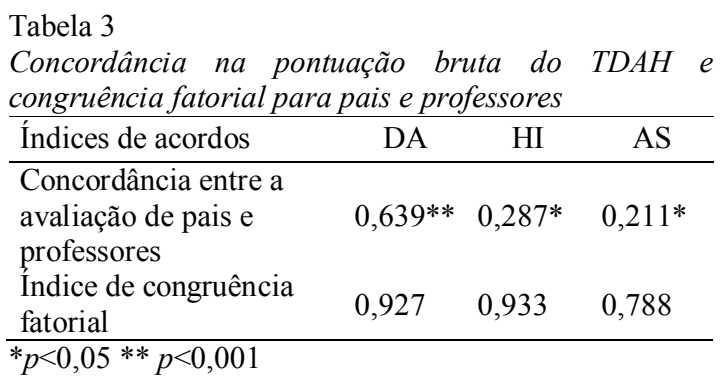

Observa-se uma maior correlação entre as pontuações dadas pelos pais e professores em Déficit de Atenção. A concordância é menor para Hiperatividade e ainda mais baixa para Comportamento Anti-social, embora todas de maneira significativa. Portanto, os pais e professores parecem avaliar de forma mais similar aspectos relacionados à cognição, como é o caso da Desatenção. O acordo diminui significativamente na avaliação de aspectos comportamentais.

\section{Discussão}

$\mathrm{Na}$ avaliação de professores e pais, a porcentagem encontrada para DA foi de 3,7\% com percentil igual ou acima de 95 , ao passo que HI e DA apresentaram um percentual de $4,7 \%$, porcentagem que corrobora a literatura nacional (Rohde, Barbosa, Tramontina, \& Polanczyk, 2000). Quando as dimensões são somadas encontra-se um percentual de $13,1 \%$. Deve-se considerar que os escores brutos de ambos os grupos de avaliadores foram transformados em uma escala percentílica a fim de compará-los.

Com respeito às variáveis que cercam as diferenças individuais, Rohde e Benczik (1999) afirmam que as características que mais modificam o transtorno são o sexo e a idade. No presente estudo, encontrou-se diferença significativa nas três escalas de TDAH a favor dos meninos, de acordo com o relato de professores. No caso, houve efeito considerável 
apenas para DA, moderado para $\mathrm{HI}$ e pequeno para $\mathrm{AS}$. Isto significa que cerca de 1 a 15\% (tamanho do efeito encontrado) das diferenças existentes entre os indivíduos dependem do fato de ser homem ou mulher.

Por outro lado, quando se analisam os resultados provenientes do relato dos pais, somente a escala de Desatenção apresenta diferença significativa em relação ao sexo da criança e, novamente, a média mais alta é a dos meninos. Nas demais escalas as médias também foram maiores, mas não de maneira estatisticamente significativa.

A maior pontuação de meninos é conhecida na literatura. Barkley (1999) afirma que os meninos teriam propensão neurológica para manifestar determinados transtornos do comportamento. Já Biederman et al. (2002) asseveram que o transtorno é mais comum entre meninos porque eles o apresentam de forma mais disruptiva que as meninas. DuPaul et al. (1998), por sua vez, apontam que, independente da dimensão, o TDAH é mais freqüente no sexo masculino. As queixas clínicas relacionadas à Hiperatividade, problemas comportamentais e de aprendizagem também mostram o mesmo padrão (Caballo $\&$ Simon, 2000). Acredita-se que causas biológicas e culturais estejam relacionadas à maior incidência de psicopatologias, principalmente a de transtornos externalizantes, em indivíduos do sexo masculino (Burns et al., 2006).

Destaca-se ainda que estudos têm buscado comprovar déficits na velocidade de processamento (Chhabildas et al., 2001) e principalmente na memória de trabalho (Barkley, 1997; Klorman et al., 1999; Oosterlaan et al., 2005). Neste estudo, os resultados da correlação entre os parâmetros medidos pelas tarefas confirmaram razoavelmente a suposição apontada pela literatura, isto é, quanto maior o escore na escala de TDAH, maior o tempo de reação e o número de erros nas tarefas, principalmente para a dimensão Desatenção (Derefinko et al., 2008).

A sub-escala de Déficit de Atenção foi a que apresentou índices mais altos e significativos de associação principalmente para professores. Mesmo em uma tarefa simples, como a de Discriminação, o tempo de decisão foi mais lento.

$\mathrm{O}$ relato de pais e professores apresentou índices de correlação mais diversos, quando se leva em conta HI e AS. Para professores, a Hiperatividade apresentou associações significativas somente para a tarefa de memória de trabalho, um resultado compatível com o encontrado por Oosterlann et al. (2005), enquanto o Comportamento Anti-social só se correlacionou com o número de erros em memória de trabalho. Acredita-se que o desempenho de crianças hiperativas em tarefas laboratoriais é significativamente mais baixo à medida que se aumenta a complexidade da tarefa (Barkley, 1997; Oosterlaan et al., 2005).

É interessante destacar que se descortina um padrão quase inverso quando a avaliação é a dos pais. A Hiperatividade se associa somente a um parâmetro da tarefa de memória, ao passo que Comportamento Anti-social se correlacionou significativamente com todas as medidas da mesma tarefa. Esse padrão conduz à conclusão que, de fato, pais e professores avaliam diferentemente as dificuldades comportamentais. Nesse sentido, professores e pais concordaram de forma moderada na avaliação de DA (uma dimensão cognitiva), enquanto foi baixa a concordância na avaliação de HI e AS (dimensões comportamentais). A raiz do problema pode estar em que a escala TDAH, especificamente aqueles itens que avaliam HI e AS, parece ter significado um tanto diferente para pais e professores. O Índice de Congruência Fatorial assim parece revelar. Acreditase, nesse sentido, que isto se deve ao fato do comportamento depender do contexto e variar conforme os diferentes grupos de amigos com os quais a criança interage (DuPaul, 2003; DuPaul et al., 1998).

Esta investigação buscou responder alguns questionamentos que, nos últimos anos, têm sido recorrentes na literatura do TDAH como prevalência, diferenças individuais, procedimentos avaliativos que pretendem confirmar sintomas em contextos diversos e identificar a relação entre TDAH e desempenho cognitivo.

Com relação à prevalência de hiperatividade, o índice encontrado na presente pesquisa (cerca de 5\%) mostrou que em uma sala de aula com 30 alunos cerca de dois deles apresentarão características do TDAH que podem vir a ser confirmadas em um psicodiagnóstico. Portanto, os professores devem estar aptos a identificar esses comportamentos para que a criança seja indicada a uma avaliação e para que sejam traçadas estratégias para lidar com os sintomas em sala de aula.

No que concerne às diferenças individuais de gênero, podese concluir, com segurança, que os indivíduos do sexo masculino apresentam médias definitivamente mais altas e possuem mais chance de apresentar o transtorno. Obviamente, outras causas estão relacionadas a este resultado, mas, em situação de psicodiagnóstico, o sexo deve ser considerado em primeira instância, uma vez que os meninos parecem demonstrar maior propensão à manifestação do TDAH.

Nos últimos dez anos, a utilização de provas cognitivas computadorizadas tem sido ampliada, a fim de se confirmar os sintomas do TDAH. Os índices de associação encontrados nesta investigação revelaram-se moderados e significativos, principalmente quando se leva em conta a avaliação dos professores. Pode-se concluir que as características do transtorno são relacionadas a um processamento cognitivo básico mais lento e também a um déficit evidente em memória de trabalho. Provavelmente essa relação pode-se estender ao campo da inteligência geral considerando-se a positiva relação entre parâmetros de processamento cognitivo e testes psicométricos de inteligência (Flores-Mendoza et al., 2001). Assim, parece válido lançar mão desse tipo de instrumento para complementar a avaliação cognitiva da criança com suspeita de TDAH.

Uma limitação do estudo, contudo, deve ser levantada: a maior parte da amostra foi constituída de sujeitos com escores médios, portanto, sem sintomas significativos para TDAH. Dessa forma, as conclusões a respeito do processamento cognitivo podem não ser generalizáveis para crianças com o transtorno. Propõe-se para estudos futuros, uma ampliação do número de sujeitos com percentil alto em TDAH, para que se tenham dados mais conclusivos a respeito dos prejuízos dos sintomas.

Por fim, pode-se afirmar que os dados encontrados, a partir de uma perspectiva dimensional, auxiliam no esclarecimento de 
algumas dúvidas recorrentes na literatura nacional do TDAH, como prevalência e diferenças individuais. Concomitantemente, o estudo trouxe implicações teóricas importantes, principalmente no que diz respeito à relação das características do transtorno com o desempenho e cognitivo do sujeito.

\section{Referências}

American Psychiatric Association. (2000). Diagnostic and statistical manual of mental disorders: DSM-IV-TR (4th ed.). Washington, DC: Author.

Associação Brasileira de Empresas de Pesquisa (2003). Critério de Classificação Econômica Brasil - CCEB - Dados com base no levantamento socioeconômico 2000-IBOPE. Obtido em 12 de março de 2004, de http// www.abep.org.

Ávila, C., Cuenca, I., Felix, V., Parcet, M-A., \& Miranda, A. (2004). Measuring impulsivity in school-aged boys and examining its relationship with ADHD and ODD ratings. Journal of Abnormal Child Psychology, 32, 295-305.

Barkley, R. A. (1997). Behavioral inhibition, sustained attention, and executive functions: constructing a unifying theory of ADHD. Psychological Bulletin, 121, 65-94.

Barkley, R. A. (1999). Attention-deficit/hyperactivity disorder. Scientific American, 279, 66-71.

Barkley, R. A. (2002). Transtorno do Déficit de Atenção/Hiperatividade-TDAH - Guia completo para pais, professores e profissionais da saúde. Porto Alegre: Artes Médicas.

Benczik, E. B. P. (2000). Manual da Escala de Transtorno do Déficit de Atenção e Hiperatividade: versão para professores. São Paulo: Casa do Psicólogo.

Biederman, J., Mick, E., Faraone, S. V., Braaten, E., Doyle, A., Spencer, T., ... Johnson, M. A. (2002). Influence of gender on attention deficit hyperactivity disorder in children referred to a psychiatric clinic. American Journal of Psychiatry, 159, 36-42.

Burns, G. L., Walsh, J. A., Gomez, R., \& Hafetz, N. (2006). Measurement and structural invariance of parents ratings of ADHD and ODD across gender for American and Malaysian children. Psychological Assessment, 18, 452-457.

Caballo, V. E., \& Simon, M. (2000). Manual de psicología clinica infantil e del adolescente - transtornos generales. Madrid: Ediciones Pirámide.

Chhabildas, N., Pennington, B. F., \& Willcutt, E. G. (2001). A comparison of the neuropsychological profiles of the DSM-IV subtypes of ADHD. Journal of Abnormal Child Psychology, 29, 529-541.

Crystal, D. S., Ostrander, R., Chen, R. S., \& August, G. J. (2001). Multimethod assessment of psychopathology among DSM-IV subtypes of children with attention-deficit/hyperactivity disorder: self-, parent, and teacher reports. Journal of Abnormal Child Psychology, 29, 189-207.

Derefinko, K. J., Adams, Z. W., Milich, R., Fillmore, M. T., Lorch, E. P., \& Lynam, D. R. (2008). Response style differences in the Inattentive and Combined subtypes of Attention Deficit/Hyperactivity Disorder. Journal of Abnormal Psychology, 36, 745-758.

Detterman, D. K., Mayer, J. D., Caruso, D. R., Legree, P. J., Conners, F. A., \& Taylor, R. (1992). Assessment of basic cognitive abilities in relation to cognitive deficits. American Journal of Mental Retardation, 97, 252-286.

DuPaul, G. (2003). Assessment of ADHD symptoms: comments on Gomez et al. Psychological Assessment, 15, 115-117.

DuPaul, G., Anastopoulos, A. D., Power, T. J., Reid, R., Ikeda, M. J., \& McGoey, K. E. (1998). Parent ratings of attention-deficit/hyperactivity disorder symptoms: factor structure and normative data. Journal of Psychopathology and Behavioral Assessment, 20, 83-102.

Flores-Mendoza, C. E. (1999). Processamento cognitivo básico e inteligência em deficientes mentais. Tese de doutorado, Universidade de São Paulo, São Paulo.

Flores-Mendoza, C. E., Colom, R. B., Garcia, L. F., \& Castilho, A. V. (2001). Dificultades en el rendimiento escolar y la memoria de trabajo. Boletim de Psicologia, 1, 21-36.

Homer, C. J., Baltz, R. D., Hickson, G. B., Miles, P. V., Newman, T. B., Shook, J. E. et al. (2000). Clinical practice guideline: diagnosis and evaluation of the child with attention-deficit/hyperactivity disorder. American Academy of Pediatrics, 105, 1158-1170.

Huang-Pollock, C. L., Higg, J. T., \& Carr, T. C. (2005). Deficient attention is hard to find: applying the perceptual load model of selective attention deficit hyperactivity disorder subtypes. Journal of Child Psychology and Psychiatry, 46, 1211-1218.

Jensen, A. (1998). The g factor. London: Praeger.

Klorman, R., Hazel-Fernandez, L. A., Shaywitz, S. E., Fletcher, J. M., Marchione, K. E., Holahan, J. M. et al. (1999). Executive functions deficits are independent of oppositional defiant disorder or reading disorder. Journal of the American Academy of Child and Adolescent Psychiatry, 38, 1148-1156.

Manuzza, S., Klein, R. G., Bessler, A., Malloy, P., \& LaPadula, M. (1998). Adult psychiatric status of hyperactive boys grown up. American Journal of Psychiatry, 155, 493-498.

Manuzza, S., Klein, R. G., \& Moulton, J. L., III. (2002). Young adult outcome of children with "situational" hyperactivity: a prospective, controlled follow-up study. Journal of Abnormal Child Psychology, 30, 191-198.

Mitsis, E. M., McKay, K. E., Schultz, K. P., Newcorn, J. H., \& Halperin, J. M. (2000). Parent-teacher concordance for DSM-IV Attention-Deficit/ Hyperactivity Disorder in a clinic-referred sample. Journal of the American Academy of Child and Adolescent Psychology, 39, 308-313.

Nigg, J. T. (2001). Is ADHD a disinhibitory disorder? Psychological Bulletin, 127, 571-598.

Oosterlaan, J., Scheres, A., \& Sergeant, J. A. (2005). Which executive functioning deficits are associated with $\mathrm{AD} / \mathrm{HD}, \mathrm{ODD} / \mathrm{CD}$ and comorbid $\mathrm{AD} / \mathrm{HD}+\mathrm{ODD} /$ CD? Journal of Abnormal Child Psychology, 33, 69-85.

Powers, T. J., Andrews, T. J., Eiraldi, R. B., Doherty, B. J., Ikeda, M. J., DuPaul, G. J. et al. (1998). Evaluating attention deficit hyperactivity disorder using multiple informants: the incremental utility of combining teacher with parent reports. Psychological Assessment, 3, 250-260.

Quay, H.C. (1997). Inhibition and attention deficit hyperactivity disorder. Journal of Abnormal Child Psychology, 25, 7-13.

Rohde, L. A., Barbosa, G., Tramontina, S., \& Polanczyk, G. (2000). Transtorno de déficit de atenção/hiperatividade. Revista Brasileira de Psiquiatria, 22, 7-11.

Rohde, L. A., \& Benczik, E. (1999). TDAH - O que é? Como ajudar? Porto Alegre: Artes Médicas.

Rowland, A. S., Skipper, B., Rabiner, D. L., Umbach, D. M., Stallone. L., Campbell, R. A. et al. (2008). The shifting subtypes of ADHD: classification depends on how symptoms reports are combines. Journal of Abnormal Child Psychology, 36, 731-743.

Simonsen, B. M., \& Bullis, M. D. (2007). The effectiveness of using a multiple gating approach to discriminate among ADHD subtypes. Journal of Emotional and Behavioral Disorders, 15, 223-236.

Zucker, M., Morris, M., Ingram, S. M., Morris, R. D., \& Bakeman, R. (2002). Concordance of self- and informant ratings of adults' current and childhood attention-deficit/hyperactivity disorder symptoms. Psychological Assessment, 14, 379-389. 
Alana Augusta Concesso de Andrade, mestre em Psicologia Social pela Universidade Federal de Minas Gerais (UFMG), é doutoranda em Psicologia pela UFMG. Endereço para correspondência: Av. Antonio Carlos, 6627 FAFICH-Depto. de Psicologia-Sala 4046 Laboratório de Avaliação das Diferenças Individuais UFMG Belo Horizonte CEP: 31270-901. Telefone: (31)3409-6277.

e-mail: alana.andrade@uol.com.br

Carmen Flores-Mendoza, doutora em Psicologia do Desenvolvimento Humano pela Universidade de São Paulo, é professora associada na Universidade Federal de Minas Gerais. 